\title{
The discovery of a novel knockdown resistance (kdr) mutation A1007G in the Voltage-Gated Sodium Channel (vgsc) on Aedes aegypti (Diptera: Culicidae) from Malaysia
}

\author{
Wan Fatma Zuharah ( $\nabla$ wfatma@usm.my ) \\ Universiti Sains Malaysia \\ Maryam Sufian \\ Universiti Sains Malaysia
}

\section{Research Article}

Keywords: Aedes, Insecticide, kdr, Mosquito, Pyrethroid, Resistance, vgsc

Posted Date: November 20th, 2020

DOl: https://doi.org/10.21203/rs.3.rs-109522/v1

License: (9) (1) This work is licensed under a Creative Commons Attribution 4.0 International License.

Read Full License

Version of Record: A version of this preprint was published at Scientific Reports on March 4th, 2021. See the published version at https://doi.org/10.1038/s41598-021-84669-w. 


\title{
TITLE PAGE
}

Type of Article: Research Article

The discovery of a novel knockdown resistance $(k d r)$ mutation A1007G in the Voltage-Gated Sodium Channel (vgsc) on Aedes aegypti (Diptera: Culicidae) from Malaysia

\author{
Wan Fatma Zuharah",2*, Maryam Sufian' \\ 1 School of Biological Sciences, Universiti Sains Malaysia, 11800 Minden, Penang, \\ MALAYSIA \\ 2 Vector Control Research Unit, School of Biological Sciences, Universiti Sains Malaysia, \\ 11800 Minden, Penang, MALAYSIA
}

*Corresponding author:

Associate Professor Dr. Wan Fatma Zuharah

School of Biological Sciences,

Universiti Sains Malaysia,

11800 Minden, Penang, MALAYSIA

Phone (or Mobile) No.: +6012-5607494

Email:wfatma@usm.my 


\section{ABSTRACT}

Background: The usage of insecticide rendered the successful vector control program with the high usage of the pyrethroid. However, the intensive and extensive use of pyrethroid, causing resistance in Aedes aegypti and hampered the control program. Knockdown resistance $(k d r)$ resulting from the Voltage-Gated Sodium Channel (vgsc) is one of the mechanisms of resistance in pyrethroid group insecticide. Investigating the phenotypic statu s of Ae. aegypti mosquitoes is a lead in knowing the current resistance status and as an indicator of the genotypic resistance. In this study, we investigate the resistance in phenotypic and genotypic of Ae. aegypti with a new $k d r$ mutation point, $\mathrm{A} 1007 \mathrm{G}$ was detected.

Methods: Using the adult bioassay, we tested the phenotypic resistance from the Selangor state against $0.75 \%$ permethrin, $0.05 \%$ deltamethrin with and without the addition of PBO synergist. Permethrin-resistant and deltamethrin-resistant, including susceptible samples, were subjected to genotyping analysis on mutation point in domain II and domain III of Voltage-Gated Sodium Channel (vgsc).

Results: Adult bioassay revealed that the Ae. aegypti was highly resistance toward $0.75 \%$ permethrin and $0.05 \%$ deltamethrin. The bioassay with the presence of PBO synergist showed an increment of mortality rate, but the status of Ae. aegypti is still resistance towards both insecticides. Genotyping result showed that three common $k d r$ mutations (S989P, V1016G, and F1534C) have existed in the Ae. aegypti population. Luckily, a new mutation on $\mathrm{A} 1007 \mathrm{G}$ was also detected in this population, and this is the first time reported.

Conclusions: This study has brought a piece of information on the current resistance status in Ae. aegypti in Malaysia. The detection of new mutation point of A1007G has added the knowledge on the resistance in mosquitoes. Thus, this study will aid the decision in using the 
insecticide usage in vector control program before this invaluable insecticide rendered ineffective in killing mosquitoes.

Keywords: Aedes, Insecticide, $k d r$, Mosquito, Pyrethroid, Resistance, vgsc

\section{INTRODUCTION}

Dengue is the most important arboviral infections of humans transmitted by Aedes mosquitoes ${ }^{1}$. Aedes aegypti (Linnaeus) is extensively known as the primary vectors of dengue viruses (DENVs), with Aedes albopictus (Skuse) regarded as a secondary vector ${ }^{2}$. DENV in humans is often life-threatening and can lead to a range of clinical manifestations from mild fever to potential dengue shock syndrome in its most severity ${ }^{3}$. In Malaysia, by November 2020, 83972 cases have been reported with 120 deaths with the highest recorded in Selangor state 4 .

The use of chemical insecticides is still a key component of Aedes vector control method by targeting the adult mosquitoes either through an indoor residual spray, treated bed nets or space spraying once larval source reduction fails to reduce the population of adult mosquitoes or adults become a serious nuisance ${ }^{5,6}$. The heavy reliance on using residual insecticides such as through intensive and prolonged use have caused insecticide resistance in Aedes mosquitoes, and reduce the effectiveness of adulticide-based control programs around the world including Malaysia ${ }^{7,8,9}$.

Organochlorines, organophosphate, carbamates and pyrethroids are four major categories of insecticides used in vector control programmes. Though, pyrethroid insecticide is extensively used on insect pests in vector control or agriculture sectors worldwide due to its fast-acting and has relatively low mammalian toxicity ${ }^{10,11}$. In Malaysia, insecticides from the class pyrethroid such as permethrin and deltamethrin are also mainly used for adulticiding 
purpose in the vector control programme by the Ministry of Health. Thus, the in ten se usage of these insecticides has been reported to cause insecticide resistance of Ae. aegypti populations in Malaysia.

Insecticide resistance can derive from different mechanisms, the main ones being modifications in the target sites, and it is known as target site resistance. In general, pyrethroids function as neurotoxins that target voltage-gated sodium channels (VGSC) and interfere electronic signalling in the nervous system, which results in paralysis and death of the mosquito, an effect often referred to as "knockdown"12,13. Target site resistance in mosquitoes is related to either single or multiple mutations in target genes; for ex ample, the VGSC gene which leads to knockdown resistance $(k d r)$, mutations in the acetylcholinesterase (Ace-1) gene and GABA receptors ${ }^{14}$. However, $k d r$ is the most well-studied target site pyrethroid resistance mechanisms in insects, including disease vectors such as against $A e$. aegypti mosquitoes ${ }^{15}$ and a good predictor of the efficacy of pyrethroids is through genotyping of $k d r$ mutant alleles ${ }^{16}$.

Mutations at three codon positions of the VGSC gene (S989P, I1011M/V and V1016G/I) have been primarily reported in domain II while mutation at the position of F1534C was reported in domain III of the pyrethroid-resistant Aedes populations worldwide. In South-East Asia, the mutation in the position of F1534C and V1016G of the VSGC was reported in the pyrethroid resistant Ae. aegypti in Malaysia ${ }^{17}$. Some of these mutations, such as the F1534C have also been reported in neighbouring countries such as in Singapore ${ }^{15,18}$ and Thailand ${ }^{19}$. Whereas, mutations at I1011V and V1016I were identified in Latin American countries $^{20}$. Interestingly, four novel mutant alleles at F1269C, T1520I, F1534S, and F1534L on domain III has been found in Vietnam ${ }^{21}$, India22, and China ${ }^{23}$ respectively. In addition, a new pyrethroid mutation (V410L) in domain I have been recently detected in Brazil ${ }^{24}$. 
Thus, it is very likely for the emergence of new $k d r$ mutations when pyrethroids remained to be the primary insecticide-based interventions in the control of Ae. aegypti. These detections act as a crucial role in resistance management of Ae. aegypti by observing the occurrence of pyrethroid resistance. By understanding the mechanisms of resistance, appropriate insecticides can be used for effective control of target vector species. In this study, we investigated the susceptibility of Ae. aegypti on the pyrethroid resistance and examined the $k d r$ mutations in this mosquito species at dengue endemic areas in Selangor, Malaysia. To our knowledge, this study reports the presence of a novel mutation A1007G in Aedes aegypti Selangor strain is reported for the first time.

\section{METHODS}

\section{Mosquito strains}

The sampling site was conducted in Selangor, which is at the centre of Peninsular Malaysia. Mosquito samples were collected from the urban area located at Puncak Perdana $\left(3^{\circ} 07^{\prime} 57.3^{\prime \prime} \mathrm{N} 101^{\circ} 29^{\prime} 35.0^{\prime \prime} \mathrm{E}\right)$ UiTM campus consisted of a university student hostel. This hostel at Puncak Perdana, Selangor is surrounded by greenery and with plenty of undeveloped lands and on-going developments. These sampling sites were chosen because the number of dengue cases is highly reported by a previous study 25 .

\section{Collection and maintenance of Aedes mosquitoes}

Sample collection was done by using ovitraps to collect eggs and larvae of mosquitoes. The traps were placed in location close to student's residential premises, and also near vegetation to collect both Aedes species. Approximately 50 ovitraps were rand omly set up for each sampling locations and were collected after five days introduced to the field. All water containing larvae and wooden paddles with eggs were brought back to the laboratory at 
Medical Entomology Laboratory, Universiti Sains Malaysia to be rear as parent generation, $\mathrm{F}_{0}$. Mosquito populations were maintained at a temperature of $25 \pm 2{ }^{\circ} \mathrm{C}$ and relative humidity $(\mathrm{RH})$ of $52 \pm 2 \%$.

\section{Permethrin and deltamethrin adult bioassay on insecticide susceptibility test}

Adult bioassays were carried out according to the standard WHO protocol ${ }^{26}$. All strains were tested against pyrethroid insecticides in four replicates of 25 non-blood fed female mosquito ranging from 3 to 5 days old. The insecticides used were; (i) $0.75 \%$ permethrin (type I), (ii) $0.05 \%$ deltamethrin (type II), (iii) $\mathrm{PBO}+0.75 \%$ permethrin, and (iv) PBO $+0.05 \%$ permethrin. Due to high resistance usually occurred in Aedes mosquito in Malaysia, Anopheles diagnostic dose was used in this study. Furthermore, the Aedes diagnostic dose is still tentative dose currently ${ }^{27}$. Controls were done in two replicates for each insecticide using silicone oil for pyrethroid control. While the Vevtor Control Research Unit (VCRU), Universiti Sains Malaysia susceptible strain was used as a reference for baseline susceptible study towards permethrin and deltamethrin insecticides.

Mosquitoes in the holding tubes were given one-hour acclimation time. Any damaged, injured, and dead mosquitoes were replaced with the healthy ones after the acclimation time. The mosquitoes from the holding tube were then transferred into the test tube containing treated impregnated paper. Knockdown data were then recorded every five minutes for an interval of one hour once exposed to insecticides. After the exposure period, mosquitoes were immediately transferred back into the holding tubes with $10 \%$ sucrose solution soaked cotton wools as a food source, and the mortality rate was determined after 24h. Percentage of mortality after $24 \mathrm{~h}$ exposure to insecticides in the adult bioassay test was characterized for the susceptibility status using WHO criteria where mosquitoes are 
considered as: (1) susceptible, if the percentage mortality is between 98 and $100 \%$, (2) incipient resistant if mortality is between $90-97 \%$ and (3) resistant if mortality is $<90 \% 26$.

Mosquito strains that were highly resistant to all insecticide treatments were also tested using insecticide synergist, PBO to assess the involvement of cytochrome P450s in the insecticide resistance mechanisms. In this case, both permethrin and deltamethrin were found highly resistance. Mosquitoes were pre-exposed to $4 \% \mathrm{PBO}$ for 1 hour before directly exposed to the mentioned insecticides. In parallel, controls were done by using bioassays with $\mathrm{PBO} 4 \%$ and exposing the mosquitoes to control papers before transferring to insecticide tubes. All treatments were replicated four times with two sets of control for each insecticide, with and without synergist associated. Overall, alive and dead Ae. aegypti samples from Selangor strain (of each insecticide treatment with and without synergist) from the $24 \mathrm{~h}$ recovery period was kept in $1.5 \mathrm{ml}$ microcentrifuge tube and immediately stored in $20^{\circ} \mathrm{C}$ until DNA extraction.

\section{Genomic DNA extraction}

Homogenate and mosquito DNA isolation from individual mosquitoes were prepared following GENEzol ${ }^{\mathrm{TM}}$ Reagent of Geneaid protocol, with slight modification during the extraction method on the amount of reagent used. A total of $100 \mu 1$ of GENEzol ${ }^{\mathrm{TM}}$ reagent was inserted into $1.5 \mathrm{ml}$ of microcentrifuge tube and homogenized using a sterile plastic grinder until disintegrated. The sample was then incubated for five minutes at room temperature, and transfer to a new microcentrifuge tube. Following the phase separation step, $20 \mu 1$ of chloroform was added into the sample, shake vigorously for $10 \mathrm{sec}$ and centrifuged at $14,000 \mathrm{x}$ f for $15 \mathrm{mins}$ at $4^{\circ} \mathrm{C}$ to separate the phases. The upper aqueous phase was then transferred to a new $1.5 \mathrm{ml}$ microcentrifuge tube. Briefly, during the DNA precipitation, $30 \mu \mathrm{l}$ of absolute ethanol was added into the sample, mixed by inverting the tube several times, 
incubated for $5 \mathrm{~min}$ at room temperature then centrifuged at $2,000 \mathrm{xg}$ for $5 \mathrm{~min}$ at $4^{\circ} \mathrm{C}$. Subsequently, the supernatant was then carefully removed. Afterwards, $100 \mu 1$ of $0.1 \mathrm{M}$ sodium citrate in $10 \%$ ethanol was added into the sample and incubated for $30 \mathrm{~min}$ at room temperature. The tube was occasionally inverted during incubation. The sample was then centrifuged at $2,000 \mathrm{x}$ g for $5 \mathrm{~min}$ at $4^{\circ} \mathrm{C}$, and the supernatant was removed. The above wash step was repeated once more. Then, $150 \mu \mathrm{l}$ of $70 \%$ ethanol was added into the sample and was incubated for $15 \mathrm{~min}$ at room temperature, while the tube was gently inverted occasionally. The sample was centrifuged at 2,000 $\mathrm{x}$ f for $5 \mathrm{~min}$ at $4^{\circ} \mathrm{C}$, and the supernatant was carefully removed. The DNA pellet was then air-dried for $10 \mathrm{~min}$ at room temperature. During DNA resuspension step, $30 \mu 1$ of TE Buffer was added to the DNA pellet. The DNA sample was then incubated at $60^{\circ} \mathrm{C}$ for $15 \mathrm{~min}$ and centrifuged at $14,000 \mathrm{x}$ g for $15 \mathrm{mins}$ at $4^{\circ} \mathrm{C} .30 \mu \mathrm{lof}$ the supernatant containing DNA was then transferred to a new $1.5 \mathrm{ml}$ microcentrifuge tube and stored at $-20^{\circ} \mathrm{C}$ until PCR analysis.

\section{Detection of knockdown resistance mutation $(k d r)$ in domain II and domain III of VGSC gene in Aedes aegypti}

To identify potential $k d r$ mutations, two fragments of the coding region of the VGSC gene spanning exon 19 to exon 31 (covering the 989, 1011, 1016, 1007 and 1534 coding positions) were amplified from DNA samples and directly sequenced. PCR mix of total $25 \mu 1$ was prepared using 12.5 $\mu$ of EconoTaq@PLUS Green 2X Master mix, $0.25 \mu 1$ of Forward primer, $0.25 \mu \mathrm{l}$ of Reverse primer, $1 \mu \mathrm{l}$ of DNA template and lastly $11 \mu \mathrm{l}$ of $\mathrm{dH} 2 \mathrm{O}$ on ice. To ensure even mixture of the PCR mix, the PCR mix was centrifuged for 10 seconds using a mini centrifuge machine before subjected to PCR analysis.

A total of two sets of PCR mix were prepared for each DNA sample, (i) for domain II (mutations at S989P, I1011M/V, L1014F, V1016G/I, and A1007G) the initial fragment 
amplification was performed by using primers AaSCF1 (AGACAATGTGGATCGCTTCC) and AaSCR4 (GGACGCAATCTGGCTTGTTA) ${ }^{28}$, while (ii) for domain III primers AaSCF7 (GAGAACTCGCCGATGAACTT) and AaSCR7 (GACGACGAAATCGAACAGGT) were used in the polymerase chain reaction to detect F1534C mutation ${ }^{28}$. The PCR parameters were set at $94^{\circ} \mathrm{C}$ for 5 mins for initial denaturation, 35 cycles of $94^{\circ} \mathrm{C}$ for $30 \mathrm{sec}$ for denaturation, $57^{\circ} \mathrm{C}$ for $30 \mathrm{sec}$ for annealing and $72^{\circ} \mathrm{C}$ for $1 \mathrm{~min}$ for exten sion, followed by final elongation step at $72^{\circ} \mathrm{C}$ for 10 mins and was set to hold at $4^{\circ} \mathrm{C}$ for $\infty 18,28$.

The PCR products were size separated on a $1.5 \%$ agarose gel stained with nucleic acid (HealthviewTM). The gel containing size separated PCR products were visualized by using a gel documentation system by BioRad (Gel DocTM XR+System). The PCR products were prepared and sent for purification and sequencing to MyTACG DNA Sequencing Services. The DNA sequencing was carried out by using primers AaSCF3 (GTGGAACTTCACCGACTTCA) and AaSCR6 (CGACTTGATCCAGTTTGGAGA) for domain II and AaSCR8 (TAGCTTTCAGCGGCTTCTTC) for domain III of Ae. aegypti ${ }^{28}$.

\section{Statistical Analysis}

The statistical analysis was done by using probit analysis from IBM SPSS Statistic Version 24 to find the knockdown time of $50 \%$ and $95 \%$ of the tested population (KdT50 and KdT95). Based on the KdT50 and KdT95 values, the resistance ratio (RR) was calculated as in the formula below:

$$
R R=\frac{K d T 50 / 95 \text { of the field strain }}{\frac{K d T 50}{95} \text { of the susceptible strain }}
$$

Based on the RR value, the field population is considered as susceptible when the RR $<5$, mosquitoes are considered to have a moderate resistance when RR is between 5 and 10 , and the mosquitoes are highly resistant when $R R>10^{26}$. 
In contrast, the percentage of mortality of the tested population was recorded after $24 \mathrm{~h}$ by using the formula as follows:

$$
\text { Observed mortality }=\frac{\text { Total number of dead mosquitoes }}{\text { Total sample size }} \times 100
$$

Abbott's formula was used as the corrective formula if the mortality rate of control replicates ranged between $10-20 \%$. However, the test was discarded and repeated if the percentage in control mortality was more than $\geq 20 \%$. Abbott's formula was not applied in this study.

$$
\text { Corrected mortality }=\frac{\% \text { Observed mortality }-\% \text { Control mortality }}{100-\% \text { Control mortality }} \times 100
$$

The sequences obtained from the sequencing company (My TAGC DNA Sequencing Services) were aligned using ClustalW and translated into a protein sequence by using protein translation tool from Mega $v 7.0$ software ${ }^{29}$.

\section{RESULTS}

\section{Susceptibility status and resistance pattern of Aedes aegypti in Selangor, Malaysia}

The results of the adult insecticide bioassays conducted on the WHO lethal dose of $0.75 \%$ permethrin for Ae. aegypti population after the one-hour test indicated that Selangor strain exhibited the highest $\mathrm{KdT}_{50}(932.97 \mathrm{~min})$ and $\mathrm{KdT}_{95}(87871.68 \mathrm{~min})$ when exposed to permethrin with the $R_{50}$ at 58.93 fold and $R R_{95}$ at 4045.66 fold respectively compared to $0.05 \%$ deltamethrin. The exposure of Ae. aegypti towards $0.05 \%$ deltamethrin showed significantly lower $\mathrm{KdT}_{50}(252.21 \mathrm{~min})$ and $\mathrm{KdT}_{95}(2305.89 \mathrm{~min})$ with $\mathrm{RR}_{50}$ value of 13.64 fold and $\mathrm{RR}_{95} 90.00$ fold respectively. This suggested that Ae. aegypti Selangor strain has 
developed significantly higher resistance towards $0.75 \%$ permethrin in comparison to $0.05 \%$ deltamethrin (Table 1).

Interestingly, the exposure of Ae. aegypti with insecticide and PBO is significantly lower compared to treatment without PBO (Table 1$)$. The $0.75 \%$ permethrin + PBO treatment indicates significantly lower $\mathrm{KdT}_{50}(129.11 \mathrm{~min})$ and $\mathrm{KdT}_{95}(888.93)$ with $\mathrm{RR}$ values of 8.15 fold and 40.93 fold respectively as compared to $0.75 \%$ permethrin treatment only. While, the RR of Ae. aegypti with $0.05 \%$ deltamethrin $+\mathrm{PBO}$ was with $\mathrm{RR}_{50}$ value of 2.82 fold and $\mathrm{RR}_{95} 4.44$ fold. This suggested that PBO might play a role in the resistance status of $A e$. aegypti population in Selangor.

The $24 \mathrm{~h}$ mortality results had confirmed that the susceptible VCRU lab strain as reference strain is still fully susceptible to both $0.75 \%$ permethrin and $0.05 \%$ deltamethrin insecticides with $100 \%$ mortality (Table 2). On the other hand, Selangor strain is strongly resistant towards both type I and type II pyrethroids as defined by WHO; < $90 \%$ mortality indicates resistance (WHO, 2016). Surprisingly, a high resistance level could be observed for Ae. aegypti towards deltamethrin $0.05 \%$ with only $13 \%$ mortality after $24 \mathrm{~h}$ exposure period. In contrast, $27 \%$ mortality was observed on Ae. aegypti when exposed to permethrin $0.75 \%$ $24 \mathrm{~h}$ post-exposure mortality thus also indicates resistance (Table 2).

For the synergist assay, pre-exposure to $\mathrm{PBO}$ induced a partial recovery of susceptibility for both deltamethrin and permethrin insecticides with $49 \%$ and $41 \%$ mortality in Ae. aegypti but still within the resistance range (Table 2). Overall, an increase in mortality was observed towards both insecticides with synergist when compared between assays without synergist suggested that cytochrome P450 monooxygenases are playing a predominant role in the resistance of Ae. aegypti population. 
DNA sequenced of the partial domain II and domain III of the VGSC gene of Ae. aegypti Selangor population revealed the presence of four mutations at segment 6 of domain II and domain III. Three mutations were present in domain II particularly at the position of S989P and V1016G which were due to the substitution on the first codon ( $\underline{T C C}$ to $\underline{\text { CCC }) \text { and }}$

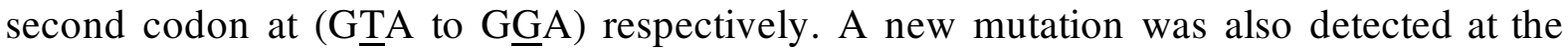

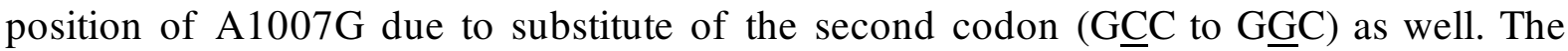
mutation A1007G is the first reported novel mutation of domain II found in Ae. aegypti mosquito population in this study that resistance toward pyrethroid represents by Alanine

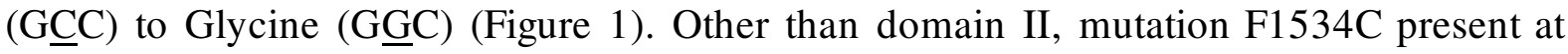
domain III was due to $\mathrm{T}>\mathrm{C}$ substitution on the second substitution of the codon, leading to Phenylalanine (TTC) to Cysteine (TGC) mutation also found in this study.

In the course of study, a total of 26 selected Ae. aegypti samples that confirmed resistance in bioassay study were sequenced for partial domain II and domain III. A total of none individuals were homozygous susceptible S989 (encoded as S/S), 17 were homozygous resistant P989 (encoded as P/P), nine were homozygous susceptible V1016 (encoded as V/V), 17 were homozygous resistant G1016 (encoded as G/G), four were heterozy gous A1007G (encoded as A/G), one was homozygous resistant G1007 (encoded as G/G), 17 were homozygous susceptible F1534 (encoded as F/F), and nine were homozygous resistant C1534 (encoded as $\mathrm{C} / \mathrm{C}$ ) which were found present in the sequences (Table 3).

Overall, we detected three patterns of co-occurrence of point mutations; S989P/V1016G, A1007G/F1534C, and V1016G/A1007G/F1534C in Ae. aegypti strain. Based on Table 3, the frequency of the mutation S989P for permethrin-resistant individuals was the highest with 0.8 . Besides, the permethrin-resistant individual has the lowest frequency of 0.2 mutant alleles 1534C. Meanwhile, deltamethrin-resistant individuals had similar frequencies of one for the mutant allele 989P and 1016G. No $k d r$ resistance alleles in 
positions 1007 and 1534 were detected in deltamethrin-resistant individuals (Figure 2). Besides, permethrin-susceptible and deltamethrin-susceptible individuals were not selected for analysis due to the low sample size and poor DNA quality of the extracted individuals. Hence, only the best PCR product and the best sequence quality were used for the analysis.

Permethrin-resistant strains with PBO synergist showed the highest frequencies of 0.6 for $1016 \mathrm{G}$ and $1534 \mathrm{C}$ mutant alleles, while with the lowest frequency of 0.2 mutant allele 1534C for deltamethrin-resistant with PBO synergist. For the susceptible strains with PBO synergist, zero frequencies of mutant $989 \mathrm{P}$ and $1016 \mathrm{G}$ were detected for permethrin. Meanwhile, similar frequencies of 0.67 mutant alleles 989P and $1016 \mathrm{G}$ were found for the deltamethrin with PBO synergist individuals (Table 3).

\section{DISCUSSION}

The development of pyrethroid resistance in the Ae. aegypti population has a potential in the failure of mosquito vector management. Although the insecticide is a primary component for the mosquito management, the unplanned and excessive used of it had led to the development of insecticide resistance ${ }^{30,31}$. A recent study has mapped the existence of resistance in Ae. aegypti on the main hotspot area in Selangor, Malaysia and highlighted the role of target site mutation profile in response to pyrethroid class (permethrin and deltamethrin).

Aedes aegypti in this study was found highly resistance towards both permethrin and deltamethrin. Even though the presence of synergist, which is Piperonyl butoxide (PBO) is not helping to reduce the resistance status in Ae. aegypti in this study. Synergist PBO is used to suppresses the activity of detoxifying enzymes such as P450s and non-specific esterases that were found in pyrethroid resistance. Even after the suppression of the detoxifying enzyme, our results still exhibited resistance on pyrethroid. Five years back, the study showed the closest area to the study sites, Kuala Lumpur was moderate resistance towards permethrin 
and deltamethrin (87\% and $89 \%$ mortality $)^{17}$. Since the Ae. aegypti in Selangor was collected in the urban setting area, the selection pressure to insecticides is higher due to the high case of dengue via fogging ${ }^{32}$ or using household insecticides such as aerosol spray ${ }^{33}$. A pyrethroid is known as a "knockdown" insecticide that paralyzed the insect which responsible in the rection of sensitivity in insect's nervous system, and the confirmed mortality can be observed after 24 hours. With the mortality of $27 \%$ (permethrin) and $13 \%$ (deltamethrin), the alive individual has developed resistance.

Generally, the four main insecticides have been used in vector management: organophosphate (OP), Organochlorine (OC), Carbamates (C) and pyrethroid (PY). Currently, OP and PY were applied for the residual spraying and space spraying, and only a limited number of insecticides can be targeted the adult stage of mosquitoes ${ }^{34}$. No new other insecticide has been approved since the last 15 years ${ }^{34}$ and more resistance has been recorded recently either on the $k d r$ mutation or resistance enzyme.

Genotyping of the $k d r$ mutation in domain II (S989, I1011, L1014 and V1016) and domain III (F1534) were highly reported in Ae. aegypti worldwide ${ }^{16,19}$. Other than these common resistance mutations, V1023G has previously reported in Indonesia and Malay sia and has played a role in the reduction of sensitivity in VGSC ${ }^{31}$. Finding from this recent study, it clearly demonstrated a new detected mutation at the position $\mathrm{A} 1007 \mathrm{G}$ in Ae. aegypti. This obviously can cause more concern on the vector control program and have found distributed in Ae. aegypti in Malaysia.

In this study, we also detected the presence up to two to three mutations in one individual. The $1016 \mathrm{G}$ mutation is usually found with a 989P mutation as found by Srisawat et al. $(2010)^{35}$. There are no proven to associate the S989P alone with the relation to pyrethroid resistance but coupling with other mutation sites can cause slightly synergistic 
effects and increase the resistance effects ${ }^{36}$. The study by Ishak $(2015)^{17}$ stated that the role of the V1016G mutation in Malaysia was revealed by the increased resistance in the Ae. aegypti that possessing the $1534 \mathrm{C}$ allele. However, the presence of A1007G coupling with F1534C resistance, or triple mutations (V1016G/A1007G/F1534C) were found in both deltamethrin and permethrin resistance samples. This mutation believed to reduce the VGSC sensitivity to both type I and type II pyrethroid due to the resistance phenotype represented in the study. In the presence of more than one mutation points together with $\mathrm{V} 1007 \mathrm{G}$, we unable to conclude that this mutation point has caused the resistance, but able to increase the resistance effects to Ae. aegypti mosquitoes. In addition, A1007G was found heterozygous, but the rest of the mutations were detected as homozygous resistance. The homozygous resistance is associate with the fitness cost of haplotypic, which survived at a higher rate as compared to heterozygous mosquitoes ${ }^{37}$.

According to a study by Ahbi et al. (2020) ${ }^{38}$, high detection of F1534C mutation in Ae. aegypti was found closely related to pyrethroid resistance found in Malaysia, and her study concludes that domain III is the major point of mutations. The predominance of a resistance haplotype of F1534C in the spawning of Ae. aegypti supported the resistance of pyrethroid. This mutation point was also detected in Ae. albopictus in Singapore ${ }^{18}$. Thus, pyrethroid resistance might occur due to the presence of $k d r$ mutation or the $\mathrm{P} 450$ monooxygenase metabolic resistance ${ }^{39}$.

The existence of a new $k d r$ mutation gene in dengue vector may work as a warning sign of overused pyrethroid in the vector control program. It is crucial to screen the current susceptibility status of Aedes mosquitoes, especially the population in the dengue areas before this invaluable insecticide is rendered ineffective in killing the Ae. aegypti. Monitoring 
on the resistance status of mosquitoes will aid on the decision of insecticide usage in the vector control program.

\section{ACKNOWLEDGEMENTS}

This project was funded under the Fundamental Research Grant Scheme, The Ministry of Higher Education Malaysia (203/PBIOLOGI/6711629) and Research University Grant under Universiti Sains Malaysia (1001/PBIOLOGI/8011066). We would like to acknowledge Assoc. Prof. Nazri Che Dom, Dr. Erni Rozaini Sulaiman and COMBI Team UiTM Puncak Alam, Selangor for their help during sampling. Furthermore, special thanks to Dr. OP Singh from NIMR New Delhi for confirming the nucleotide sequences.

\section{DECLARATIONS}

WFZ - Funding, design framework, conception, supervision, interpretation of data, writing review \& editing

MS-conception, design of the work, analysis, interpretation of data, writing - original draft Conflict of interest: Each author has approved the submitted version (and any substantially modified version that involves the author's contribution to the study) and have agreed both to be personally accountable for the author's own contributions. The funders did not have any additional role in the study design, data collection and analysis, decision to publish, or preparation of the manuscript.

Ethical approval: No ethical approval is needed for this project

\section{REFERENCES}

1. Cameron PS, Jeremy JF, Nguyen V, Bridget W. Dengue. The New England Journal of Medicin, 2012; 366(1): 1423-1432. 
2. Whitehorn J, Kien DT, Nguyen NM, Nguyen HL, Kyrylos PP, Carrington LB, et al. Comparative susceptibility of Aedes albopictus and Aedes aegypti to dengue virus infection after feeding on blood of viremic humans: Implications for public health. J Infect Dis 2005; 212:1182-1190.

3. World Health Organization. Dengue: Guidelines for Diagnosis, Treatment, Prevention and Control 2019; WHO/HTM/NTD/DEN/2009.1.

4. Idengue. Available at https://idengue.mysa.gov.my/. Accessed on 15 November 2020

5. Farajollahi A, Healy SP, Unlu I, Gaugler R, Fonseca DM. Effectiveness of ultra-low volume nighttime applications of an adulticide against diurnal Aedes albopictus, a critical vector of dengue and chikungunya viruses. PLoS One 2012; 7: e49181.

6. Karunamoorthi K, Sabesan S, Jegajeevanram K, Vijayalakshmi J. Role of traditional antimalarial plants in the battle against the global malaria burden. Vector Borne Zoonotic Dis 2013;13(8):521-44.

7. Chen XB, Liu XC, Zhou L, Liu2 ZL. Essential oil composition and larvicidal activity of Clinopodium gracile (Benth) Matsum (Labiatae) aerial parts against the Aedes albopictus mosquito. Trop J Pharm Res 2013; 12 (5): 799-804.

8. Maciel-De-Freitas R, Marques WA, Peres RC, Cunha SP, Lourenço De Oliveira R. Variation in Aedes aegypti (Diptera: Culicidae) container productivity in a slum and a suburban district of Rio de Janeiro during dry and wet seasons. Mem Inst Oswaldo Cruz, Rio de Janeiro 2017; 102 .

9. Othman WN, Ahmad NW, Lim LH, Dhang CC, Mustapha WNW, Hadi AA, Azirun MS. Detection of permethrin resistance in Aedes albopictus Skuse, collected from Titiwang Zone, Kuala Lumpur, Malaysia. Proc ASEAN Congr Trop Med Parasitol 2008; 3:69-77.

10. Housset P, Dickmann R. A promise fulfilled-pyrethroid development and the benefits for agriculture and human health. Bayer CropScience 2009; 62:135-44.

11. Ranson H, N'guessan R, Lines J, Moiroux N, Nkuni Z, Corbel V. Pyrethroid resistance in African anopheline mosquitoes: what are the implications for malaria control? Trends Parasitol. 2011;27(2):91-8.

12. Narahashi T. Neuronal ion channels as the target sites of insecticides. Pharmacol Toxicol. 1996; 79(1):1-14.

13. Brito LP, Linss JG, Lima-Camara TN, Belinato TA, Peixoto AA, Lima JBP, et al. Assessing the effects of Aedes aegypti kdr mutations on pyrethroid resistance and its fitness cost. PLoS One 2013; 8(4):60878.

14. Hemingway J, Ranson $\mathrm{H}$. Insecticide resistance in insect vectors of human disease. Annu Rev Entomol. 2010; 45:371-91.

15. Kawada H, Higa Y, Komagata2 O, Kasai S , Tomita T, Yen NT , et al. Widespread distribution of a newly found point mutation in voltage-gated sodium channel in pyrethroid-Resistant Aedes aegypti populations in Vietnam. PLoS Negl Trop Dis 2004; 3(10): e0000527

16. Li CX, Kaufman PE, Xue RD, Zhao MH, Wang G, Yan T, et al. Relationship between insecticide resistance and $k d r$ mutations in the dengue vector Aedes aegypti in Southern China. Parasit. Vectors 2015; 8: 325. 
17. Ishak IH, JaalZ, Ranson H, Wondji CS. Contrasting patterns of insecticide resistance and knockdown resistance (kdr) in the dengue vectors Aedes aegypti and Aedes albopictus from Malaysia. Parasit Vectors 2015; 8:181.

18. Kasai S, Ng LC, Lam-Phua SG, Tang CS, Itokawa K, Komagata O, et al. First detection of a putative knockdown resistance gene in major mosquito vector, Aedes albopictus. Jpn J Infect Dis. 2011;64(3):217-21.

19. Yanola J, Somboon P, Walton C, Nachaiwieng W, Somwang P, Prapatadara L . Highthroughput assays for detection of the F1534C mutation in the voltage-gated sodium channel gene in permethrin-resistant Aedes aegypti and the distribution of this mutation throughout Thailand. Trop. Med. Int. Health 2011; 16: 501-509.

20. Saavedra-Rodriguez K, Urdaneta-Marquez L, Rajatileka S, Moulton M, Flores AE, Fernandez-Salas I, et al. A mutation in the voltage-gated sodium channel gene associated with pyrethroid resistance in Latin American Aedes aegypti. Insect Mol Biol. 2007; 16:785-98.

21. Kawada H, Higa Y, Komagata O, Kasai S, Tomita T, Yen NT, et al. Widespread distribution of a newly found point mutation in voltage-gated sodium channel in pyrethroid-resistant Aedes aegypti populations in Vietnam. PLoS Negl. Trop. Dis. 2009; 3(10): e527.

22. Kushwah RB, Dykes CL, Kapoor N, Adak T, Singh OP. Pyrethroid-resistance and presence of two knockdown resistance (kdr) mutations, F1534C and a novel mutation T1520I, in Indian Aedes aegypti. PLoS Negl Trop Dis 2015; 9: e3332

23. Chen H, Li K, Wang X, Yang X. Lin Y, Cai F, et al. First identification of kdr allele F1534S in VGSC gene and its association with resistance to pyrethroid insecticides in Aedes albopictus populations. Infect Dis Poverty 2016; 5:31

24. Haddi K, Tomé HVV 1, Du Y, Valbon WR, Nomura Y, Martins GF, et al. Detection of a new pyrethroid resistance mutation (V410L) in the sodium channel of Aedes aegypti: a potential challenge for mosquito control. Sci Rep 2017; 19(7): 46549.

25. Madzlan F, Dom NC, Zakaria N, Hasnan SNA, Tiong CS, Camalxaman SN. Profiling of dengue vectors breeding habitat at urban residential areas in Shah Alam, Malaysia. Serangga 2017; 22(2): 185-216.

26. World Health Organization. Test procedures for insecticide resistance monitoring in malaria vector mosquitoes - 2nd ed. 2016; 56p

27. World Health Organization Monitoring and managing insecticide resistance in Aedes mosquito populations. 2016; WHO/ZIKV/VC/16.1.13pp

28. Kawada H, Higa Y, Futami K, Muranami Y, Kawashima E, Osei JH, et al. Discovery of point mutations in the voltage-gated sodium channel from African Aedes aegypti populations: Potential phylogenetic reasons for gene introgression. PLoS Negl Trop Dis. 2016;10: e0004780.

29. Kumar S, Stecher G, Tamura K. MEGA7: Molecular Evolutionary Genetics Analysis Version 7.0 for Bigger Dataset. Mol Biol Evol 2016; 33(7):1870-4.

30. Rosilawati R, Lee HL, Nazni WA, Nurulhusna AH, Roziah A, KhairulAsuad M, et al. Pyrethroid resistance status of Aedes (Stegomyia) aegypti (Linneaus) from dengue endemic areas in Peninsular Malaysia. Int Med J Malays 2017; 16:73-78. 
31. Wuliandari JR, Lee SF, White VL, Tantowijoyo W, Hoffmann AA, EndersbyHarshman NM. Association between Three Mutations, F1565C, V1023G and S996P, in the Voltage-Sensitive Sodium Channel Gene and Knockdown Resistance in Aedes aegypti from Yogyakarta, Indonesia.. Insects 2015; 6(3):658-85.

32. Ang KT, Satwant S. Epidemiology and new Initiatives in the prevention and control of dengue in Malaysia. Dengue Bullet 2001; 25:7-14.

33. Chen CD, Nazni WA, Lee HL, Sofian-Azirun M. Susceptibility of Aedes aegypti and Aedes albopictus to temephos in four study sites in Kuala Lumpur City Center and Selangor State. Malaysia Trop Biomed 2005; 22(2):207-16.

34. World Health Organization. Global Insecticide Use for Vector-Borne Disease Control-A 10-Year Assessment (2000-2009) 5th ed. World Health Organization; Geneva, Switzerland 2011.

35. Srisawat R, Komalamisra N, Eshita Y, Zheng M, Ono K, Itoh TQ, et al. Point mutations in domain II of the voltage-gated sodium channel gene in deltamethrinresistant Aedes aegypti (Diptera: Culicidae). Appl Entomol Zool 2010; 45: 275-282.

36. Hirata K, Komagata O, Itokawa K, Yamamoto A, Tomita T, Kasai S. A single crossing-over event in voltage-gated sodium channel gene may cause critical failure of dengue mosquito control by insecticide. PLoS Negl. Trop. Dis. 2014; 8: e3085.

37. Stenhouse SA, Plernsub S, Yanola J, Lumjuan N, Dantrakool A, Choochote W, et al. Detection of the V1016G mutation in the voltage-gated sodium channel gene of Aedes aegypti (Diptera: Culicidae) by allele-specific PCR assay, and its distribution and effect on deltamethrin resistance in Thailand. Parasit Vectors. 2013; 6(1):253.

38. AhbiRami R, Ishak IH, Yahya ZS, Zuharah WF. Knockdown resistance (kdr) in dengue vectors, Aedes aegypti and Aedes albopictus from Malaysia: A post-flood risk assessment. Gen Mol Biol 2020; 19 (2): gmr18604

39. Smith LB, Kasai S and Scott JG. Pyrethroid resistance in Aedes aegypti and Aedes albopictus: Important mosquito vectors of human diseases. Pestic Biochem Phys 2016; 133: 1-12. 

Table 1. Knockdown time ( $\mathrm{min}) \mathrm{KdT}_{50}$ and $\mathrm{KdT}_{95}$ of one-hour exposure time towards two different types of WHO insecticide treatments (permethrin $0.75 \%$ and deltamethrin $0.05 \%$ ) and PBO synergist against Aedes aegypti female adults for susceptible VCRU lab strain and Selangor field strain.

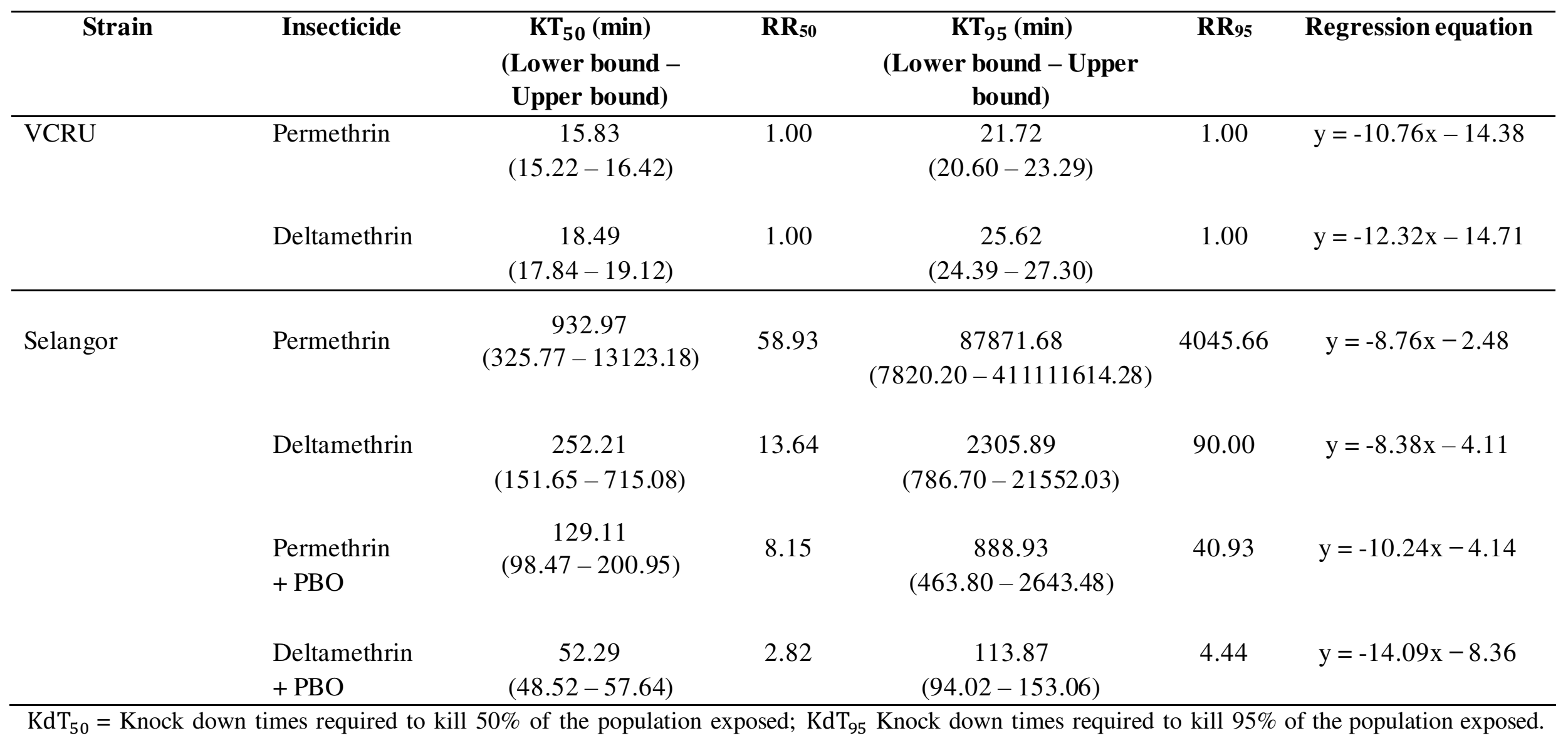


Table 2. Resistance status of Aedes aegypti female adults after $24 \mathrm{~h}$ exposure period towards two different types of WHO insecticide treatments (permethrin $0.75 \%$ and deltamethrin $0.05 \%$ ) and PBO synergist for susceptible VCRU lab strain and Selangor field strain.

\begin{tabular}{llcl}
\hline Strain & Insecticide & $\begin{array}{c}\text { \% Mortality } \pm \text { SE } \\
(\mathbf{n})\end{array}$ & Status \\
\hline \multirow{2}{*}{ VCRU } & Permethrin & $100 \pm 0(100)$ & $\mathrm{S}$ \\
& Deltamethrin & $100 \pm 0(100)$ & $\mathrm{S}$ \\
\hline \multirow{2}{*}{ Selangor } & Permethrin & $27 \pm 1.44(100)$ & $\mathrm{R}$ \\
& Deltamethrin & $13 \pm 0.85(100)$ & $\mathrm{R}$ \\
& Permethrin + PBO & $41 \pm 2.87(100)$ & $\mathrm{R}$ \\
& Deltamethrin + PBO & $49 \pm 1.31(100)$ & $\mathrm{R}$
\end{tabular}

$\mathrm{S}$ indicated susceptible and $\mathrm{R}$ indicated resistance. 
Table 3. Frequencies of S989P, V1016G, A1007G and F1534C substitution in parental of the VSGC gene in Aedes aegypti Selangor strain.

\begin{tabular}{|c|c|c|c|c|c|c|c|c|c|c|c|c|c|c|c|c|c|c|}
\hline \multirow[t]{3}{*}{ Insecticide } & \multirow[t]{3}{*}{$\mathrm{N}$} & \multirow[t]{3}{*}{ Phenotype } & \multicolumn{12}{|c|}{ Genotype } & \multicolumn{4}{|c|}{ Resistance allelic frequency } \\
\hline & & & \multicolumn{3}{|c|}{ S989P } & \multicolumn{3}{|c|}{ V1016G } & \multicolumn{3}{|c|}{$\mathrm{A} 1007 \mathrm{G}$} & \multicolumn{3}{|c|}{ F1534C } & \multirow[b]{2}{*}{$\mathrm{S} / \mathrm{P}$} & \multirow[b]{2}{*}{$\mathrm{V} / \mathrm{G}$} & \multirow[b]{2}{*}{$\mathrm{A} / \mathrm{G}$} & \multirow[b]{2}{*}{$\mathrm{F} / \mathrm{C}$} \\
\hline & & & SS & SP & $\mathrm{PP}$ & VV & VG & GG & AA & $\mathrm{AG}$ & GG & $\mathrm{FF}$ & $\mathrm{FC}$ & $\mathrm{CC}$ & & & & \\
\hline \multirow[t]{2}{*}{ Permethrin } & 5 & $\mathrm{R}$ & $\begin{array}{l}1 \\
(0.2)\end{array}$ & 0 & $\begin{array}{l}4 \\
(0.8)\end{array}$ & $\begin{array}{l}2 \\
(0.4)\end{array}$ & 0 & $\begin{array}{l}3 \\
(0.6)\end{array}$ & $\begin{array}{l}5 \\
(1)\end{array}$ & 0 & 0 & $\begin{array}{l}4 \\
(0.8)\end{array}$ & 0 & $\begin{array}{l}1 \\
(0.2)\end{array}$ & 0.8 & 0.6 & 0 & 0.2 \\
\hline & 3 & $\mathrm{~S}$ & - & - & - & - & - & - & - & - & - & - & - & - & - & - & - & - \\
\hline \multirow[t]{2}{*}{ Deltamethrin } & 5 & $\mathrm{R}$ & 0 & 0 & $\begin{array}{l}5 \\
(1)\end{array}$ & 0 & 0 & $\begin{array}{l}5 \\
(1)\end{array}$ & $\begin{array}{l}5 \\
(1)\end{array}$ & 0 & 0 & $\begin{array}{l}5 \\
(1)\end{array}$ & 0 & 0 & 1 & 1 & 0 & 0 \\
\hline & 3 & $S$ & - & - & - & - & - & - & - & - & - & - & - & - & - & - & - & - \\
\hline \multirow[t]{2}{*}{$\begin{array}{l}\text { Permethrin } \\
+\mathrm{PBO}\end{array}$} & 5 & $\mathrm{R}$ & $\begin{array}{l}3 \\
(0.6)\end{array}$ & 0 & $\begin{array}{l}2 \\
(0.4)\end{array}$ & $\begin{array}{l}2 \\
(0.4)\end{array}$ & 0 & $\begin{array}{l}3 \\
(0.6)\end{array}$ & $\begin{array}{l}3 \\
(0.6)\end{array}$ & $\begin{array}{l}2 \\
(0.4)\end{array}$ & 0 & $\begin{array}{l}2 \\
(0.4)\end{array}$ & 0 & $\begin{array}{l}3 \\
(0.6)\end{array}$ & 0.4 & 0.6 & 0.2 & 0.6 \\
\hline & 3 & $\mathrm{~S}$ & $\begin{array}{l}3 \\
(1)\end{array}$ & 0 & 0 & $\begin{array}{l}3 \\
(1)\end{array}$ & 0 & 0 & $\begin{array}{l}1 \\
(0.3)\end{array}$ & $\begin{array}{l}2 \\
(0.67)\end{array}$ & 0 & 0 & 0 & $\begin{array}{l}3 \\
(1)\end{array}$ & 0 & 0 & 0.34 & 1 \\
\hline \multirow[t]{2}{*}{$\begin{array}{l}\text { Deltamethrin } \\
+\mathrm{PBO}\end{array}$} & 5 & $\mathrm{R}$ & $\begin{array}{l}1 \\
(0.2)\end{array}$ & 0 & $\begin{array}{l}4 \\
(0.8)\end{array}$ & $\begin{array}{l}1 \\
(0.2)\end{array}$ & 0 & $\begin{array}{l}4 \\
(0.8)\end{array}$ & $\begin{array}{l}4 \\
(0.8)\end{array}$ & 0 & $\begin{array}{l}1 \\
(0.2)\end{array}$ & $\begin{array}{l}4 \\
(0.8)\end{array}$ & 0 & $\begin{array}{l}1 \\
(0.2)\end{array}$ & 0.8 & 0.8 & 0.3 & 0.2 \\
\hline & 3 & $\mathrm{~S}$ & $\begin{array}{l}1 \\
(0.3)\end{array}$ & 0 & $\begin{array}{l}2 \\
(0.67)\end{array}$ & $\begin{array}{l}1 \\
(0.33)\end{array}$ & 0 & $\begin{array}{l}2 \\
(0.67)\end{array}$ & $\begin{array}{l}3 \\
(1)\end{array}$ & 0 & 0 & $\begin{array}{l}2 \\
(0.67)\end{array}$ & 0 & $\begin{array}{l}1 \\
(0.33)\end{array}$ & 0.67 & 0.67 & 0 & 0.33 \\
\hline
\end{tabular}



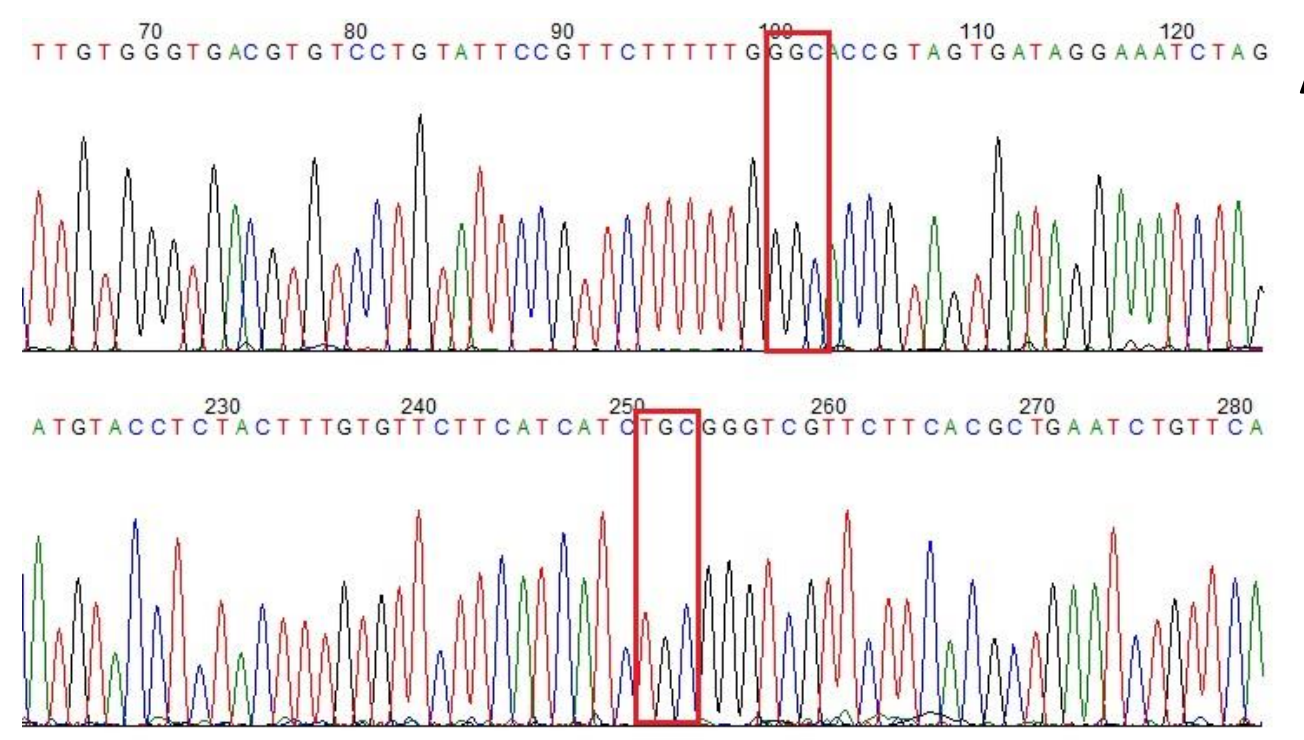

B
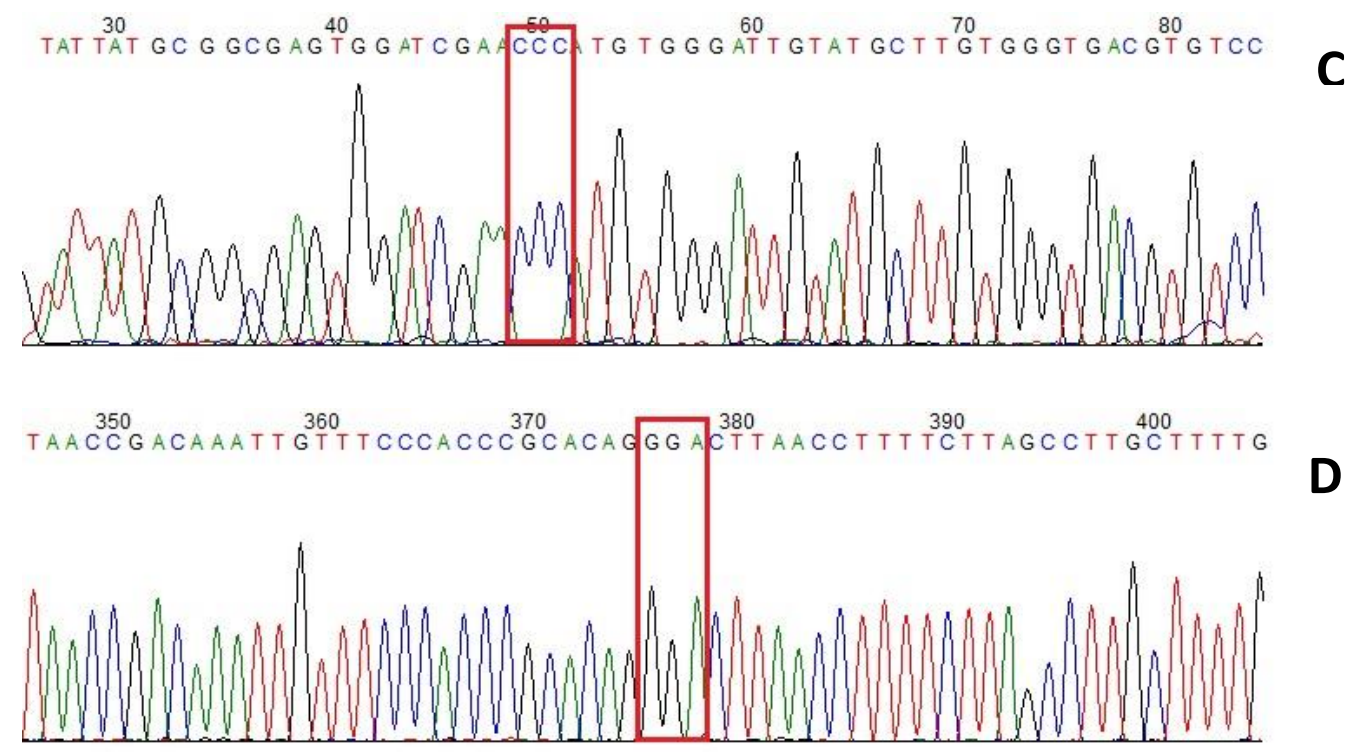

Figure 1: Chomotograh of sequences at a position of homozygous (A) S989P, (B) V1016G, (C) A1007G (a new mutation) and (D) F1534C. A red box indicated the position of the amino acids. 

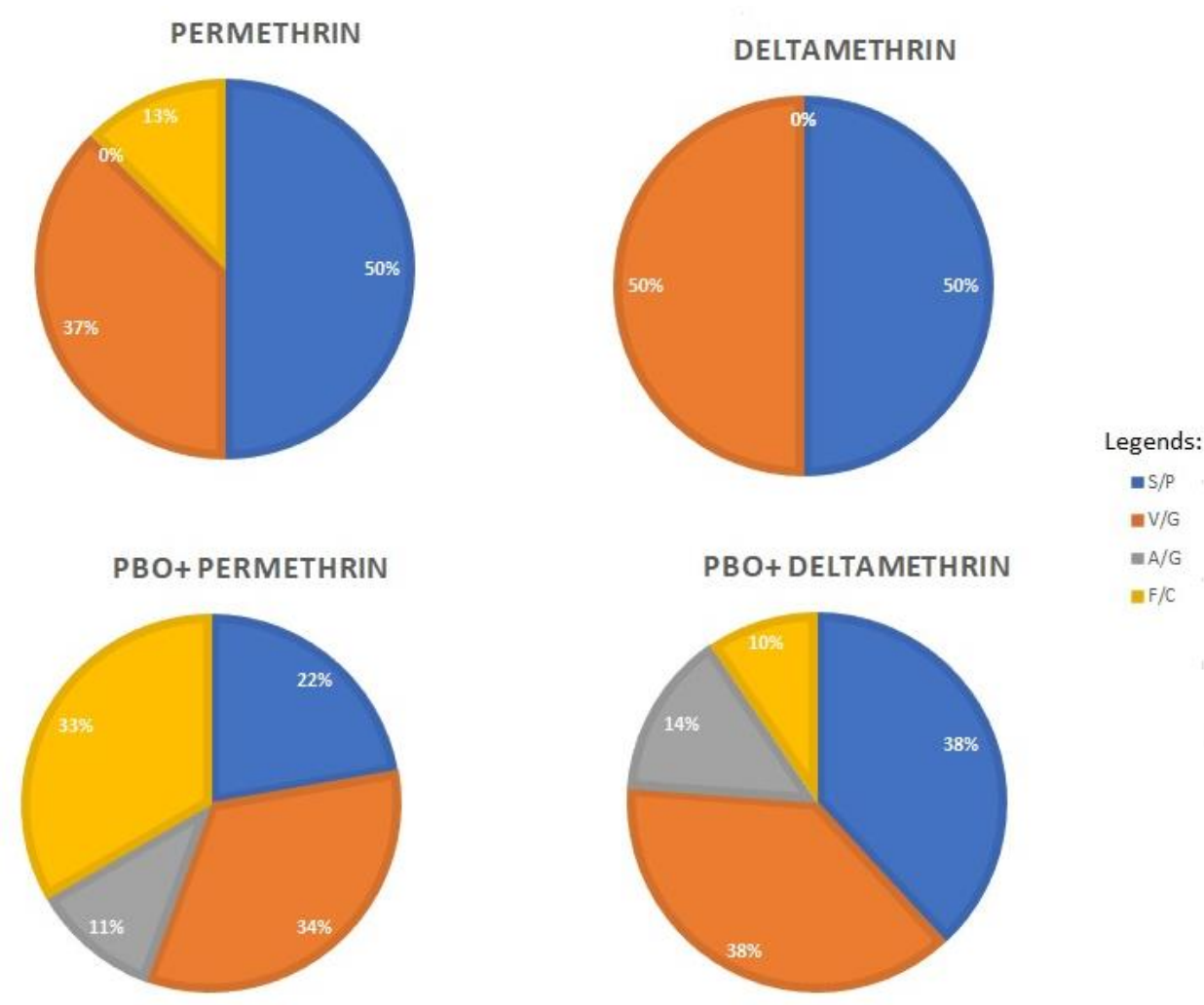

Figure 2: The resistance allelic frequency of Aedes aegypti Selangor strain identified with amino acid substitution in domain II VGSC 


\section{Figures}
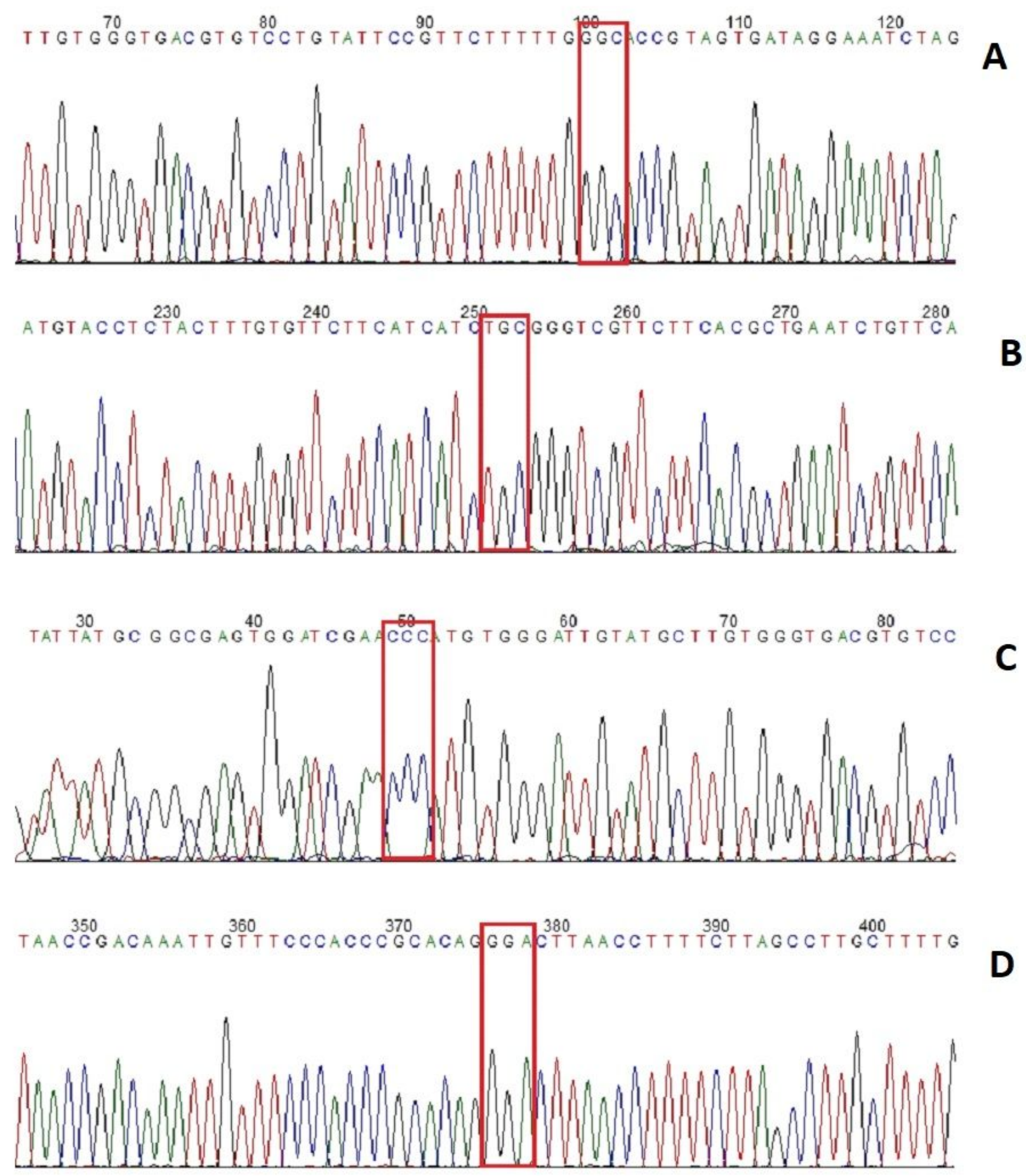

\section{Figure 1}

Chomotograh of sequences at a position of homozygous (A) S989P, (B) V1016G, (C) A1007G (a new mutation) and (D) F1534C. A red box indicated the position of the amino acids. 

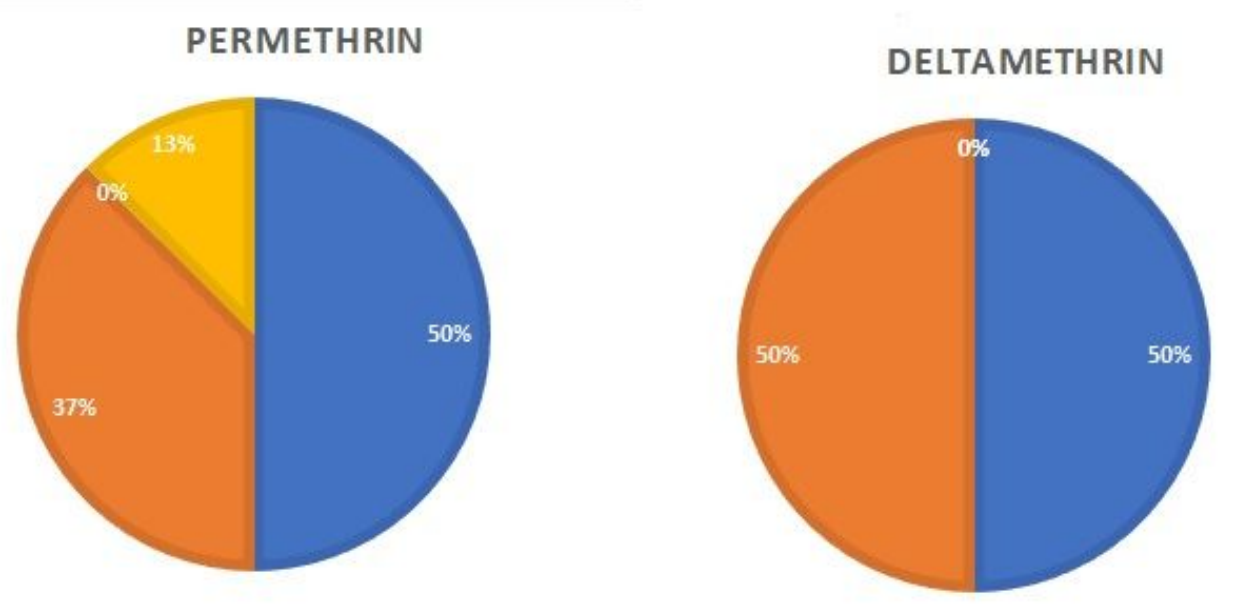

$$
\begin{aligned}
& \text { Legends: } \\
& \mathrm{m} / \mathrm{P} \\
& \text { a } / \mathrm{G}
\end{aligned}
$$

PBO+ PERMETHRIN
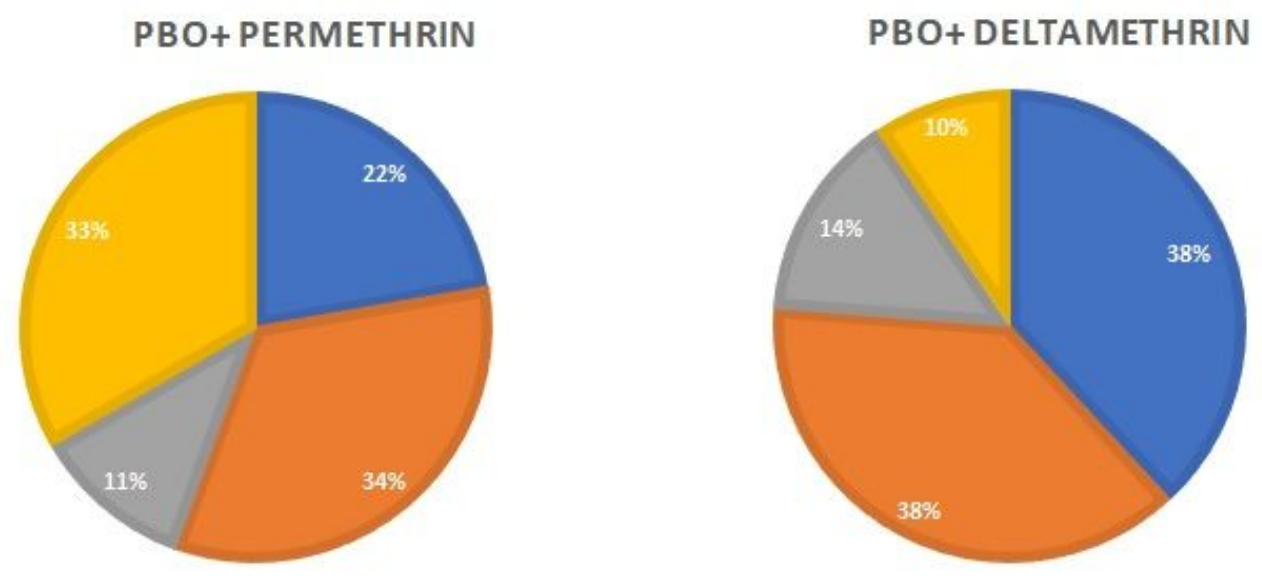

\section{Figure 2}

The resistance allelic frequency of Aedes aegypti Selangor strain identified with amino acid substitution in domain II VGSC 